\title{
Outage Performance Analysis of Multicarrier Relay Selection for Cooperative Networks
}

\author{
Shuping Dang, Justin P. Coon, Gaojie Chen and David E. Simmons \\ Department of Engineering Science, University of Oxford, Oxford, UK, OX1 3PJ \\ Email: \{shuping.dang, justin.coon, gaojie.chen, david.simmons\} @eng.ox.ac.uk
}

\begin{abstract}
In this paper, we analyze the outage performance of two multicarrier relay selection schemes, i.e. bulk and persubcarrier selections, for two-hop orthogonal frequency-division multiplexing (OFDM) systems. To provide a comprehensive analysis, three forwarding protocols: decode-and-forward (DF), fixedgain (FG) amplify-and-forward (AF) and variable-gain (VG) AF relay systems are considered. We obtain closed-form approximations for the outage probability and closed-form expressions for the asymptotic outage probability in the high signal-to-noise ratio (SNR) region for all cases. Our analysis is verified by Monte Carlo simulations, and provides an analytical framework for multicarrier systems with relay selection.
\end{abstract}

Keywords-Multicarrier relay selection, parallel fading channel, cooperative systems, outage performance.

\section{INTRODUCTION}

Cooperative communications and relay-assisted systems have attracted a large amount of attention since they were proposed and comprehensively analyzed in [1]. In such a network, relays are employed as intermediate communication nodes to assist the transmission between source and destination, so that the communications can be maintained when the direct transmission link between source and destination undergoes deep fading [2], [3]. It has been proved that with the help of relays and proper forwarding protocols, the network coverage expansion, energy efficiency, system reliability and quality of service (QoS) can be significantly enhanced [4][7]. In particular, relay selection can further enhance the system performance and obtain an extra diversity [8]. A variety of selection schemes have been proposed and analyzed for different types of relays and channel conditions [9]-[14].

Meanwhile, multicarrier communications, especially orthogonal frequency-division multiplexing (OFDM), is also proposed and utilized in relay-assisted networks, which is capable of providing a better system performance and bandwidth efficiency over frequency selective channels [15], [16]. But relay selection in multicarrier systems is not straightforward, because this is a two-tier selection/allocation problem involving relay selection and subcarrier allocation. In [17], two relay selection schemes for OFDM systems, i.e. bulk selection (a single relay is selected for transmission on all subcarriers) and per-subcarrier selection (selection is treated independently for each subcarrier, thus, potentially, leading to transmission via multiple relays) are proposed and analyzed.

However, all previous works on multicarrier relay selection are carried out based on an assumption that the outage con- dition regarding each subcarrier is treated individually, which corresponds to the block fading channel model when a user is allocated a contiguous block of carriers that lie within a coherence bandwidth. To be more realistic, we should consider the parallel fading channel model that general OFDM systems are well approximated by. The parallel fading channel model is utilized to model a fading channel consisting of a finite number of flat independent and identically distributed (i.i.d.) fading subchannels [18]. The most unique property of the parallel fading channel relative to the conventional block fading channel is the definition of outage probability. Considering the coding over the parallel fading channel, the outage probability is defined as the probability that the mutual information of all subchannels is smaller than a target transmission rate [19]. However, the mathematical tractability of the outage probability over the parallel fading channel is rather poor, because the distribution of the summation of a finite number of random variables cannot be derived in a generic closed-form expression [20]. In [21], an oversimplified case of a parallel fading channel with only two subchannels is analyzed and an integral formula for the outage probability is given. Also, upper and lower bounds as well as an approximation for the outage probability for any number of subchannels are determined in [18] and [22], respectively.

On the other hand, to the best of the authors' knowledge, the work considering multicarrier relay selection for twohop OFDM systems approximated by parallel fading channel model has not been fully treated. To fill this gap, we analyze this scenario in a Rayleigh fading condition. Note, the method applied in this paper can be easily tailored to analyze other channel conditions.

The main contributions of this paper are summarized infra:

- We obtain closed-form generic approximations for outage probabilities of two-hop OFDM systems when applying bulk and per-subcarrier relay selection schemes.

- We derive closed-form approximations for the outage probability of decode-and-forward (DF), fixedgain (FG) amplify-and-forward (AF) and variable-gain (VG) AF relay systems with bulk and per-subcarrier relay selection schemes.

- We obtain closed-form asymptotic expressions for the outage probability with different selection schemes at high SNR and also derive diversity gains. 
The rest of this paper is organized as follows. In Section II. the system model and fundamentals are given. Then, the outage performance is analyzed in Section III Specific applications including DF, FG AF and VG AF relay systems are investigated in Section IV. The analysis is numerically verified by Monte Carlo simulations and further discussed in Section V] Finally, Section VI concludes the paper.

\section{SySTEM MODEL}

\section{A. Parallel fading channel and outage probability}

In this paper, we consider a two-hop OFDM system with $K$ orthogonal subcarriers and $M$ relays gathered in a relay cluster, the size of which is relatively small compared to the distance between source and destination. Therefore, $K$ parallel fading subchannels are constructed at each hop, and for each subcarrier, there are $M$ interfering channels. We denote the sets of subcarriers and relays as $\mathcal{K}=\{1,2, \ldots, K\}$ and $\mathcal{M}=\{1,2, \ldots, M\}$. Also, we assume the entire two-hop OFDM system operates in a half-duplex protocol and the direct transmission link between source and destination does not exist due to deep fading. As a result, two orthogonal time slots are required for one complete transmission from source to destination via relay(s). Here, we denote i.i.d. Rayleigh channel coefficients in the first and second hops by $h_{1}(m, k)$ and $h_{2}(m, k) \forall m \in \mathcal{M}$ and $k \in \mathcal{K}$. The channel gain $\left|h_{i}(m, k)\right|^{2}$, where $i \in\{1,2\}$, is exponentially distributed with the mean of $\mu_{i}$. Therefore, its probability density function (PDF) and cumulative distribution function (CDF) are

$$
f_{\left|h_{i}\right|^{2}}(x)=e^{-x / \mu_{i}} / \mu_{i} \Leftrightarrow F_{\left|h_{i}\right|^{2}}(x)=1-e^{-x / \mu_{i}} .
$$

Subsequently, the end-to-end SNR transmitted on the $k$ th subcarrier and forwarded by the $m$ th relay is denoted as $\gamma(m, k)$. Accordingly, the outage probability over the parallel fading channel can be defined as

$$
P_{\text {out }}(s)=\mathbb{P}\left\{\sum_{k=1}^{K} \ln \left(1+\gamma\left(m_{k}, k\right)\right)<s\right\}
$$

where $\mathbb{P}\{\cdot\}$ denotes the probability of the enclosed; $m_{k}$ is the index of the relay selected to forward the $k$ th subcarrier; we also let $s=2 \xi$ for the convenience of the following analysis, where $\xi$ is the mutual information outage threshold 1 In addition, we assume all noise statistics are assumed to be zero-mean, complex Gaussian random variables with variance $N_{0} / 2$ per dimension, from which the noise power can be expressed by $N_{0}$.

\section{B. Forwarding protocols}

We assume equal bit and power allocation schemes are applied and the average transmit power per subcarrier at source and relay is the same, denoted by $P_{t}$. Therefore, the instantaneous end-to-end SNR of the $k$ th subcarrier forwarded by the $m$ th relay using a DF protocol is written as

$$
\gamma_{D F}(m, k)=\bar{\gamma} \min \left(\left|h_{1}(m, k)\right|^{2},\left|h_{2}(m, k)\right|^{2}\right),
$$

where $\bar{\gamma}=P_{t} / N_{0}$. Similarly, for FG AF relaying that is blind to all channel conditions and is only able to amplify the

\footnotetext{
${ }^{1}$ The factor ' 2 ' comes from the fact that two time slots are required for one complete transmission in two-hop systems.
}
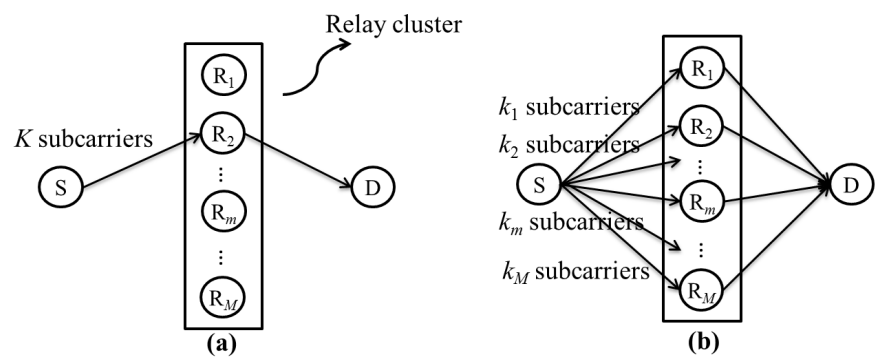

Fig. 1. Illustration of (a) bulk, (b) per-subcarrier relay selection schemes for single source, single destination and $M$ clustered relays with $K$ subcarriers. Note, $K=\sum_{m=1}^{M} k_{m}$ for $k_{m} \in \mathbb{N}, \forall 1 \leq m \leq M$.

received signal by a fixed gain, the instantaneous end-to-end SNR is written as

$$
\gamma_{F G}(m, k)=\frac{\bar{\gamma}^{2}\left|h_{1}(m, k)\right|^{2}\left|h_{2}(m, k)\right|^{2}}{\bar{\gamma} \mu_{1}+\bar{\gamma}\left|h_{2}(m, k)\right|^{2}+1} .
$$

For VG AF relaying which is able to estimate channel conditions and amplify accordingly, the instantaneous end-to-end SNR is thereby

$$
\gamma_{V G}(m, k)=\frac{\bar{\gamma}^{2}\left|h_{1}(m, k)\right|^{2}\left|h_{2}(m, k)\right|^{2}}{\bar{\gamma}\left|h_{1}(m, k)\right|^{2}+\bar{\gamma}\left|h_{2}(m, k)\right|^{2} P_{t}+1} .
$$

\section{Relay selection schemes}

1) Bulk selection: By the bulk selection scheme, the source only selects one out of $M$ relays by which all subcarriers are forwarded according to the selection criterion

$$
\mathcal{L}^{\text {bulk }}=\arg \max _{m \in \mathcal{M}}\left\{\sum_{k=1}^{K} \ln (1+\gamma(m, k))\right\},
$$

where $\mathcal{L}^{b u l k}$ is the set (of cardinality one) denoting the only selected relay. This selection scheme is easy to implement and will not involve an overcomplicated coordination protocol, because there is only one selected relay. However, it is obvious that the outage performance cannot be optimized in this case.

2) Per-subcarrier selection: The per-subcarrier selection scheme selects $L$ relays from $M$ relays in a per-subcarrier manner and $1 \leq L \leq \min (M, K)$. Therefore, the relay selected to forward the $\bar{k}$ th subcarrier is individually determined by

$$
l^{p s}(k)=\arg \max _{m \in \mathcal{M}} \ln (1+\gamma(m, k))=\arg \max _{m \in \mathcal{M}} \gamma(m, k),
$$

where $l^{p s}(k)$ is the set of the selected relay corresponding to the $k$ th subcarrier. Then, this selection process will be repeatedly applied for all subcarriers and finally $L$ relays are selected. The set of all $L$ selected relays is denoted by $\mathcal{L}^{p s}=\bigcup_{k=1}^{K}\left\{l^{p s}(k)\right\}$. Note, it is allowed that $l^{p s}(k)=l^{p s}(n)$ for $k \neq n$, i.e. one relay is allowed to assist in forwarding two or more subcarriers at the same time. Obviously, this selection scheme is optimal in terms of outage performance, but this optimality will result in a high system complexity [23]. For illustration purposes, these two selection schemes are illustrated in Fig. 1 . 


\section{Outage Performance Analysis}

\section{A. Bulk selection}

According to 2 and (6), the a posteriori outage probability with bulk selection can be defined by

$$
\begin{aligned}
& P_{\text {out }}(s)=\mathbb{P}\left\{\max _{m \in \mathcal{M}}\left\{\sum_{k=1}^{K} \ln (1+\gamma(m, k))\right\}<s\right\} \\
& =\prod_{m=1}^{M} \mathbb{P}\left\{\sum_{k=1}^{K} \ln (1+\gamma(m, k))<s\right\} \stackrel{(a)}{=}\left(F_{I}(s)\right)^{M},
\end{aligned}
$$

where $F_{I}(s):=\mathbb{P}\left\{\sum_{k=1}^{K} \ln (1+\gamma(m, k))<s\right\}, \forall m \in \mathcal{M}$ and $\forall k \in \mathcal{K}$; (a) is valid, because all parallel subchannels are assumed to be i.i.d.

Assume the CDF and PDF of $\gamma(m, k)$ are $F_{\gamma}(s)$ and $f_{\gamma}(s)$, respectively. Now following the Proposition 1 proved in [22], we also make a hypothesis that $f_{\gamma}(s)$ can be expanded as a power series about zero as

$$
f_{\gamma}(s)=s^{q}\left(g_{0}+g_{1} s+O\left(s^{2}\right)\right),
$$

where $q$ is a non-negative integer representing the inherent subchannel diversity; $g_{0}, g_{1}$ are non-zero functions of $\bar{\gamma}$ and satisfy the condition $g_{1}(\bar{\gamma})=o\left(g_{0}(\bar{\gamma})\right)$ when $\bar{\gamma} \rightarrow \infty$. This is a common assumption applicable to most two-hop channel cases [24]. Then, $F_{I}(s)$ can be written as [22]

$$
\begin{aligned}
& F_{I}(s)=\frac{S_{0}(K, s, q)^{K q+1}}{\left(S_{1}(K, s, q)-S_{0}(K, s, q)\right)^{K q}} \\
& \times f_{\gamma}\left(\frac{S_{1}(K, s, q)-S_{0}(K, s, q)}{S_{0}(K, s, q)}\right)^{K}+O\left(\frac{1}{\bar{\gamma}^{K(q+1)+2}}\right) .
\end{aligned}
$$

The coefficient $S_{x}(K, s, q)$ is defined as

$$
\begin{aligned}
& S_{x}(K, s, q):=\sum_{j=0}^{q}\left(\begin{array}{l}
q \\
j
\end{array}\right)(-1)^{j} \sum_{\substack{a_{p} \in \mathbb{N}, \forall 0 \leq p \leq q \\
\sum_{p=0}^{q} a_{p}=K-1}}\left(\begin{array}{c}
K-1 \\
a_{0}, a_{1}, \ldots, a_{q}
\end{array}\right) \\
& \times\left[\prod_{p=0}^{q}\left(\left(\begin{array}{c}
q \\
p
\end{array}\right)(-1)^{p}\right)^{a_{p}}\right] \cdot\left[\frac{1}{2 \pi i} \int_{C} \frac{e^{s z} \mathrm{~d} z}{\prod_{k=0}^{K}\left(z-\beta_{k}\right)}\right],
\end{aligned}
$$

where $\beta_{k}$ is the $k$ th element in

$\mathbf{B}=(0, \underbrace{q+1, \ldots, q+1}_{a_{0} \text { terms }}, \underbrace{q, \ldots, q}_{a_{1} \text { terms }}, \ldots, \underbrace{1, \ldots, 1}_{a_{q} \text { terms }}, q+1+x-j)$

and $C$ is the contour which encloses all poles of the integrand.

It should be noted that $\frac{1}{2 \pi i} \int_{C} \frac{e^{s z} \mathrm{~d} z}{\prod_{k=0}^{K}\left(z-\beta_{l}\right)}$ is equivalent to the inverse Laplace transform of $1 / \prod_{k=0}^{K}\left(z-\beta_{k}\right)$, which can be expressed by the closed form as follows [25]:

$$
\frac{1}{2 \pi i} \int_{C} \frac{e^{s z} \mathrm{~d} z}{\prod_{k=0}^{K}\left(z-\beta_{k}\right)}=\sum_{k=0}^{K}\left[\frac{e^{\beta_{k} s}}{\prod_{\substack{1 \leq n \leq k \\ n \neq k}}\left(\beta_{k}-\beta_{n}\right)}\right] .
$$

However, this closed-form expression given above is only valid if all poles of the integrand on the are simple (first-order poles), i.e. $\beta_{n} \neq \beta_{k}$, for $n \neq k$. Closed-form expressions exist for the cases with higher-order poles, but cannot be written as a general form. This is the reason why we still keep the integral form in (11) for generality. Another reason for keeping the integral form is because this integral form can be efficiently evaluated by computer-based simulations using the residue theorem [26].

As a result, the a posteriori outage probability by bulk selection as defined in (8) can be determined by

$$
\begin{aligned}
& P_{\text {out }}^{\text {bulk }}(s)=\left(F_{I}(s)\right)^{M}=\frac{S_{0}(K, s, q)^{M(K q+1)}}{\left(S_{1}(K, s, q)-S_{0}(K, s, q)\right)^{M K q}} \\
& \times f_{\gamma}\left(\frac{S_{1}(K, s, q)-S_{0}(K, s, q)}{S_{0}(K, s, q)}\right)^{M K}+O\left(\frac{1}{\bar{\gamma}^{M K(q+1)+2}}\right) .
\end{aligned}
$$

Furthermore, we can derive the asymptotic outage probability at high SNR by power series and obtain

$$
P_{\text {out }}^{\text {bulk }}(s) \sim \tilde{P}_{\text {out }}^{\text {bulk }}(s)=\left(S_{0}(K, s, q) g_{0}^{K}\right)^{M} .
$$

Note, because of the condition $g_{1}(\bar{\gamma})=o\left(g_{0}(\bar{\gamma})\right)$ when $\bar{\gamma} \rightarrow$ $\infty$, the diversity order can be derived from $\sqrt{15}$ by $G_{d}^{b u l k}=$ $-\lim _{\bar{\gamma} \rightarrow \infty} \frac{\log P_{\text {out }}^{\text {bulk }}(s)}{\log \bar{\gamma}}=M K(q+1)$.

\section{B. Per-subcarrier selection}

According to (2) and (7), the a posteriori outage probability when per-subcarrier selection is employed can be defined by

$$
P_{\text {out }}(s)=\mathbb{P}\left\{\sum_{k=1}^{K} \ln \left(1+\max _{m \in \mathcal{M}} \gamma(m, k)\right)<s\right\} .
$$

Denote $\Psi(m, k)=\max _{m \in \mathcal{M}} \gamma(m, k)$. We can obtain the CDF and PDF of $\Psi(m, k)$ as

$$
F_{\Psi}(s)=\left(F_{\gamma}(s)\right)^{M} \Leftrightarrow f_{\Psi}(s)=M\left(F_{\gamma}(s)\right)^{M-1} f_{\gamma}(s) .
$$

Following the assumption given in $97, f_{\Psi}(s)$ can be further expressed by

$$
\begin{aligned}
& f_{\Psi}(s)=\frac{M g_{0}^{M}}{(q+1)^{M-1}} s^{M(q+1)-1} \\
& +M\left[\frac{g_{0}^{M-1} g_{1}}{(q+1)^{M-1}}+\frac{(M-1) g_{0}^{M-1} g_{1}}{(q+1)^{M-2}(q+2)}\right] s^{M(q+1)} \\
& +O\left(s^{M(q+1)+1}\right)
\end{aligned}
$$

Therefore, if we denote

$$
\left\{\begin{array}{l}
q^{\prime}=M(q+1)-1 \\
g_{0}^{\prime}=\frac{M g_{0}^{M}}{(q+1)^{M-1}} \\
g_{1}^{\prime}=M\left[\frac{g_{0}^{M-1} g_{1}}{(q+1)^{M-1}}+\frac{(M-1) g_{0}^{M-1} g_{1}}{(q+1)^{M-2}(q+2)}\right]
\end{array}\right.
$$

$f_{\Psi}(s)$ can also be written as

$$
f_{\Psi}(s)=s^{q^{\prime}}\left(g_{0}^{\prime}+g_{1}^{\prime} s+O\left(s^{2}\right)\right),
$$

which also aligns with the form given in (9). This result indicates that we can similarly employ Proposition 1 derived in [22] to analyze the outage performance of per-subcarrier relay selection over the parallel fading channel, and the only modification is to replace $f_{\gamma}(\cdot)$ with $f_{\Psi}(\cdot)$. Hence, the outage 
performance of per-subcarrier relay selection over the parallel fading channel as defined in (16) can be calculated by

$$
\begin{aligned}
& P_{\text {out }}^{p s}(s)=\frac{S_{0}\left(K, s, q^{\prime}\right)^{K q^{\prime}+1}}{\left(S_{1}\left(K, s, q^{\prime}\right)-S_{0}\left(K, s, q^{\prime}\right)\right)^{K q^{\prime}}} \\
& \times f_{\Psi}\left(\frac{S_{1}\left(K, s, q^{\prime}\right)-S_{0}\left(K, s, q^{\prime}\right)}{S_{0}\left(K, s, q^{\prime}\right)}\right)^{K}+O\left(\frac{1}{\bar{\gamma}^{K\left(q^{\prime}+1\right)+2}}\right) .
\end{aligned}
$$

Furthermore, we can derive the asymptotic outage probability at high SNR by power series and obtain

$$
P_{\text {out }}^{p s}(s) \sim \tilde{P}_{\text {out }}^{p s}(s)=S_{0}\left(K, s, q^{\prime}\right) g_{0}^{\prime K} .
$$

Similarly to the case for bulk selection, we can also obtain the diversity of per-subcarrier selection from 22 to be $G_{d}^{p s}=$ $M K(q+1)$ as expected.

\section{APPLICATIONS}

\section{A. DF relay networks}

According to (1) and (3), we can derive the PDF of the end-to-end SNR $\gamma_{D F}(m, k)$ for DF relay networks by

$$
f_{\gamma}^{D F}(s)=\frac{1}{\bar{\gamma}}\left(\frac{1}{\mu_{1}}+\frac{1}{\mu_{2}}\right) e^{-\frac{s}{\bar{\gamma}}\left(\frac{1}{\mu_{1}}+\frac{1}{\mu_{2}}\right)},
$$

which can be expanded by power series as $\bar{\gamma} \rightarrow \infty$ to the standard expanded form given in (9), and thus can be substituted into (14) and (21) to yield the approximated outage probability with bulk and per-subcarrier selections over the parallel fading channel.

\section{B. $F G A F$ and $V G A F$ relay networks}

By (1) and (4), we can similarly derive the PDF of the endto-end SNR $\gamma_{F G}(m, k)$ for FG AF relay networks by [27]

$$
\begin{aligned}
& f_{\gamma}^{F G}(s)=e^{-\frac{s}{\bar{\gamma} \mu_{1}}}\left[\frac{2\left(1+\bar{\gamma} \mu_{1}\right)}{\bar{\gamma}^{2} \mu_{1} \mu_{2}} K_{0}\left(2 \sqrt{\frac{s\left(1+\bar{\gamma} \mu_{1}\right)}{\bar{\gamma}^{2} \mu_{1} \mu_{2}}}\right)\right. \\
& \left.+\frac{2}{\bar{\gamma} \mu_{1}} \sqrt{\frac{s\left(1+\bar{\gamma} \mu_{1}\right)}{\bar{\gamma}^{2} \mu_{1} \mu_{2}}} K_{1}\left(2 \sqrt{\frac{s\left(1+\bar{\gamma} \mu_{1}\right)}{\bar{\gamma}^{2} \mu_{1} \mu_{2}}}\right)\right],
\end{aligned}
$$

where $K_{x}(\cdot)$ is the $x$ th order modified Bessel function of the second kind.

Similarly, by (1) and (5), the PDF of the end-to-end SNR $\gamma_{V G}(m, k)$ for $\mathrm{VG}$ AF relay networks is given by [27]

$$
\begin{aligned}
& f_{\gamma}^{V G}(s)=e^{-\frac{s}{\bar{\gamma}}\left(\frac{1}{\mu_{1}}+\frac{1}{\mu_{2}}\right)}\left[\frac{2\left(1+2 s \mu_{2}\right)}{\bar{\gamma}^{2} \mu_{1} \mu_{2}} K_{0}\left(2 \sqrt{\frac{s\left(1+s \mu_{2}\right)}{\bar{\gamma}^{2} \mu_{1} \mu_{2}}}\right)\right. \\
& \left.+\frac{2}{\bar{\gamma}}\left(\frac{1}{\mu_{1}}+\frac{1}{\mu_{2}}\right) \sqrt{\frac{s\left(1+s \mu_{2}\right)}{\bar{\gamma}^{2} \mu_{1} \mu_{2}}} K_{1}\left(2 \sqrt{\frac{s\left(1+s \mu_{2}\right)}{\bar{\gamma}^{2} \mu_{1} \mu_{2}}}\right)\right]
\end{aligned}
$$

Although (24) and 25) cannot be expanded by power series to the form as given in (14), these two PDFs can still be substituted into (14) and (21) to yield the approximated outage probability with a divergent error at low SNR (see Corollary 3 in [22]).

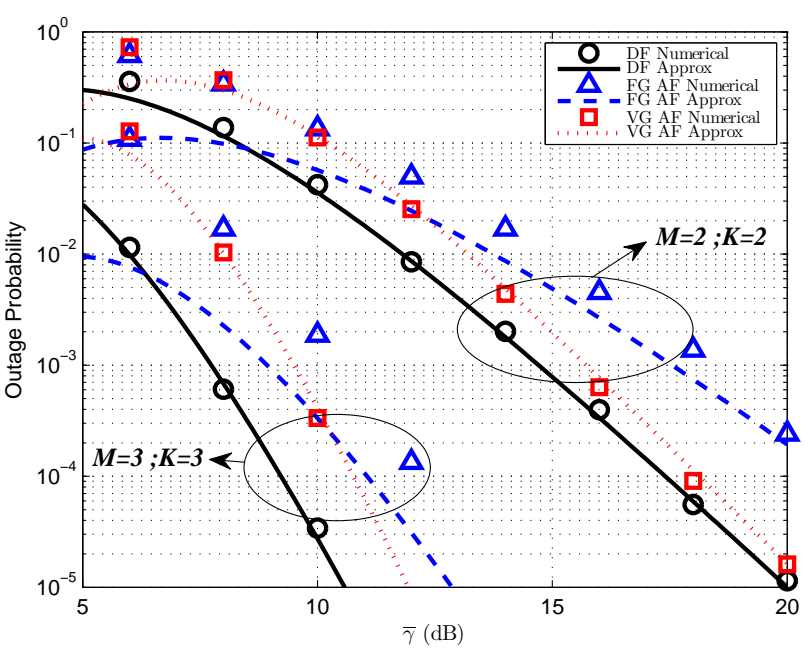

Fig. 2. Bulk selection case: numerical results and analytical approximations with different system configurations of $M$ and $K$.

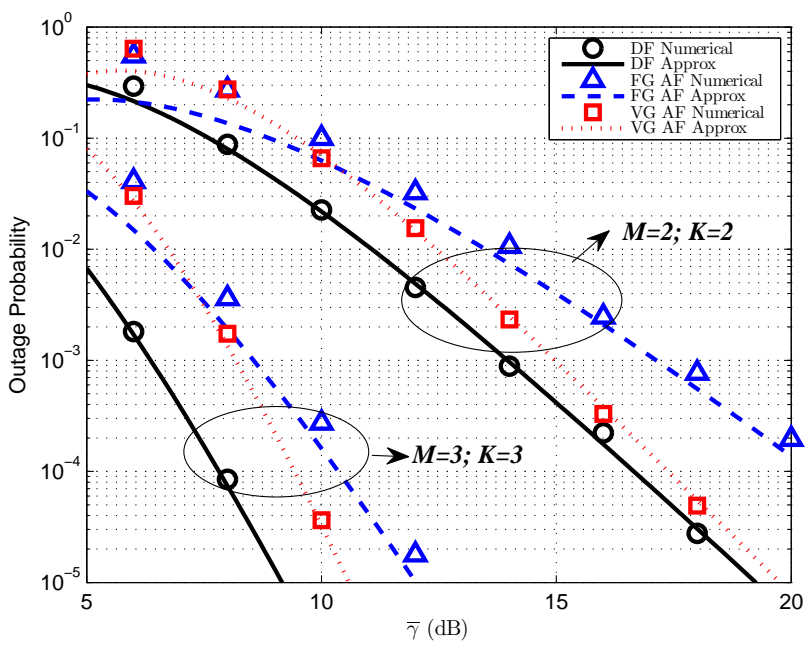

Fig. 3. Per-subcarrier selection case: numerical results and analytical approximations with different system configurations of $M$ and $K$.

\section{NumericAl Results}

To verify our analysis in Section III and Section IV we carry out Monte Carlo simulations and present the numerical results in this section. To simplify simulations, without losing generality, we normalize the two-hop system by letting $\mu_{1}=\mu_{2}=1$ and $s=2$ (i.e. $\xi=1$ as the mutual information outage threshold). Then, we can plot the relation among $\bar{\gamma}$, analytical approximations for outage probability (as given in (14) and (21)) and numerical outage probabilities for different combinations of $M$ and $K$. We present the simulation results for bulk selection and per-subcarrier selection in Fig. 2 and Fig. 3 respectively. From these two figures, it is clear that the proposed approximations for the outage probability with bulk and per-subcarrier selections over the parallel fading channel have been verified to be effective to approximate the outage performance of $\mathrm{DF}, \mathrm{FG} \mathrm{AF}$ and $\mathrm{VG}$ AF relay systems at high SNR. Note, although DF, FG AF and VG AF relay systems have the same diversity gain determined by $M K(q+1)$, the convergence rate of FG AF case to the asymptotic region is smaller due to different correction terms. Another minor point 
is that the similarity of outage performance between DF and VG AF relay systems in the high SNR region as valid over block fading channels [24], can still be found valid over the parallel fading channel. In addition, by comparing Fig. 2 and Fig. 3. we can find that the performance difference between bulk and per-subcarrier selections is not so significant as that in block fading channels [28]. This is because the outage event over the parallel fading channel depends on the mutual information over all subcarriers, instead of each individual subcarrier.

\section{CONCLUSION}

In this paper, we analyzed multicarrier relay selection for two-hop OFDM systems and obtained closed-form approximations for the outage probabilities when applying bulk and per-subcarrier relay selections. It has been numerically shown that the derived approximations for $\mathrm{DF}, \mathrm{FG} \mathrm{AF}$ and $\mathrm{VG} \mathrm{AF}$ relay networks are valid and able to effectively approximate the exact outage performance at high SNR. Meanwhile, we also derived the generic asymptotic expressions for the outage probabilities at high SNR when applying bulk and per-subcarrier relay selections. All these results provide an insight into the outage performance of multicarrier relay systems over parallel fading channels.

\section{ACKNOWLEDGMENT}

This work was supported by the SEN grant (EPSRC grant number EP/N002350/1) and the grant from China Scholarship Council (No. 201508060323).

\section{REFERENCES}

[1] J. N. Laneman, D. N. C. Tse, and G. W. Wornell, "Cooperative diversity in wireless networks: Efficient protocols and outage behavior," IEEE Transactions on Information Theory, vol. 50, no. 12, pp. 3062-3080, Dec. 2004.

[2] P. Guo, Y. Bai, Z. Ma, S. Wu, and S. Dang, "Relay technology for multi-carrier systems: A research overview," in IEEE C3IT, Hooghly, India, Feb. 2015

[3] A. Chandra, C. Bose, and M. K. Bose, "Wireless relays for next generation broadband networks," IEEE Potentials, vol. 30, no. 2, pp. 39-43, Mar. 2011.

[4] A. K. Sadek, Z. Han, and K. J. R. Liu, "Distributed relay-assignment protocols for coverage expansion in cooperative wireless networks," IEEE Transactions on Mobile Computing, vol. 9, no. 4, pp. 505-515, Apr. 2010.

[5] I. Ku, C. X. Wang, and J. Thompson, "Spectral-energy efficiency tradeoff in relay-aided cellular networks," IEEE Transactions on Wireless Communications, vol. 12, no. 10, pp. 4970-4982, Oct. 2013.

[6] H. A. Suraweera, P. J. Smith, and J. Armstrong, "Outage probability of cooperative relay networks in Nakagami-m fading channels," IEEE Communications Letters, vol. 10, no. 12, pp. 834-836, Dec. 2006.

[7] S. R. Cho, W. Choi, and K. Huang, "QoS provisioning relay selection in random relay networks," IEEE Transactions on Vehicular Technology, vol. 60, no. 6, pp. 2680-2689, July 2011.

[8] D. S. Michalopoulos and G. K. Karagiannidis, "Performance analysis of single relay selection in Rayleigh fading," IEEE Transactions on Wireless Communications, vol. 7, no. 10, pp. 3718-3724, Oct. 2008.

[9] P. L. Yeoh, M. Elkashlan, and I. B. Collings, "Selection relaying with transmit beamforming: a comparison of fixed and variable gain relaying," IEEE Transactions on Communications, vol. 59, no. 6, pp. 1720-1730, June 2011
[10] G. Chen and J. A. Chambers, "Exact outage probability analysis for cooperative af relay network with relay selection in presence of intercell interference," Electronics Letters, vol. 48, no. 21, pp. 1346-1347, Oct. 2012.

[11] I. Krikidis, "Relay selection for two-way relay channels with MABC DF: A diversity perspective," IEEE Transactions on Vehicular Technology, vol. 59, no. 9, pp. 4620-4628, Nov. 2010.

[12] Y. Wang and J. P. Coon, "Outage probability of fixed-gain dual-hop relay selection channels with heterogeneous fading," EURASIP Journal on Wireless Communications and Networking, vol. 2015, no. 1, pp. 111, 2015.

[13] G. Chen, O. Alnatouh, and J. Chambers, "Outage probability analysis for a cognitive amplify-and-forward relay network with single and multi-relay selection," IET Communications, vol. 7, no. 17, pp. 19741981, Nov. 2013.

[14] S. S. Soliman and N. C. Beaulieu, "Exact analysis of dual-hop AF maximum end-to-end SNR relay selection," IEEE Transactions on Communications, vol. 60, no. 8, pp. 2135-2145, Aug. 2012.

[15] Z. Wang and G. B. Giannakis, "Wireless multicarrier communications," IEEE Signal Processing Magazine, vol. 17, no. 3, pp. 29-48, May 2000.

[16] B. Gui, L. J. Cimini, and L. Dai, "OFDM for cooperative networking with limited channel state information," in IEEE MILCOM, Washington, DC, Oct. 2006.

[17] B. Gui, L. Dai, and L. J. C. Jr., "Selective relaying in cooperative OFDM systems: two-hop random network," in IEEE WCNC, Las Vegas, NV, Mar. 2008

[18] B. Bai, W. Chen, K. B. Letaief, and Z. Cao, "Outage exponent: A unified performance metric for parallel fading channels," IEEE Transactions on Information Theory, vol. 59, no. 3, pp. 1657-1677, Mar. 2013.

[19] D. Tse and P. Viswanath, Fundamentals of Wireless Communication, ser. Wiley series in telecommunications. Cambridge University Press, 2005.

[20] M. Sandell and J. Coon, "Performance of combined bulk and per-tone antenna selection precoding in coded ofdm systems," IEEE Transactions on Communications, vol. 60, no. 3, pp. 655-660, Mar. 2012.

[21] L. H. Ozarow, S. Shamai, and A. D. Wyner, "Information theoretic considerations for cellular mobile radio," IEEE Transactions on Vehicular Technology, vol. 43, no. 2, pp. 359-378, May 1994.

[22] J. P. Coon, D. E. Simmons, and M. D. Renzo, "Approximating the outage probability of parallel fading channels," IEEE Communications Letters, vol. 19, no. 12, pp. 2190-2193, Dec. 2015.

[23] Y. Li, W. Wang, and F. C. Zheng, "Combined bulk and per-tone relay selection in cooperative OFDM systems," in IEEE ICCC, Beijing, China, Aug. 2012.

[24] S. Dang, J. P. Coon, and G. Chen, "An equivalence principle for OFDMbased combined bulk/per-subcarrier relay selection over equally spatially correlated channels," IEEE Transactions on Vehicular Technology, 2016.

[25] A. Erdélyi, Tables of Integral Transforms. New York, NY: McGrawHill, 1954

[26] B. Davies, Integral Transforms and Their Applications, ser. Texts in Applied Mathematics. Springer New York, 2012.

[27] S. Dang, J. P. Coon, and D. E. Simmons, "Combined bulk/per-subcarrier relay selection in two-hop ofdm systems," in Proc. IEEE VTC Spring, Nanjing, China, May 2016.

[28] _ "Combined bulk and per-tone relay selection in super dense wireless networks," in IEEE ICC, London, UK, June 2015. 